\title{
Gestational Diabetes Mellitus (GDM), Diagnostics, Therapy and Follow-up Care
}

\author{
Practice recommendation - Summary of the S3 guideline (AWMF register \\ number: 057-008)
}

Authors

Ute Schäfer-Graf', Katharina Laubner², Sandra Hummel³, Ulrich Gembruch ${ }^{4}$, Tanja Groten ${ }^{5}$, Franz Kainer ${ }^{6}$, Melita Grieshop ${ }^{7}$, Dagmar Bancher-Todesca ${ }^{8}$, Mila Cervar-Zivakovic ${ }^{9}$, Irene Hösli ${ }^{10}$, Matthias Kaltheuner ${ }^{11}$, Reinhold Gellner ${ }^{12}$, Alexandra Kautzky-Willer ${ }^{13}$, Christoph Bührer ${ }^{14}$

\section{Affiliations}

1 Berlin Diabetes Center for pregnant women, Department of Gynecology and Obstetrics, St. Joseph Hospital, Berlin, Germany

2 Department of Internal Medicine II, Department of Endocrinology and Diabetology, University Hospital Freiburg, Germany

3 Institute for Diabetes Research, Research Center for Health and Environment (GmbH), Helmholtz Zentrum München, Germany

4 Department of Gynecology, Obstetrics and Prenatal Medicine, University Hospital Bonn, Germany

5 Department of Obstetrics, University Hospital Jena, Germany

6 Department of Obstetrics and Prenatal Medicine, Hallerwiese Hospital, Nuremberg, Germany

7 Protestant University of Applied Science, Berlin, Germany

8 Döbling Private Hospital, Vienna, Austria

9 Division of Obstetrics, Department of Gynecology and Obstetrics, LKH- University Hospital Graz, Austria

10 Department of Obstetrics and Gynecology, University Hospital Basel, Basel, Switzerland

11 Joint practice Dres. Kaltheuner/v. Boxberg, Leverkusen

12 Department of Gastroenterology and Hepatology, University Hospital Münster, Germany

13 Division of Endocrinology and Metabolism, Department of Internal Medicine III, Medical University of Vienna, Austria

14 Department of Neonatology, Charité - Universitätsmedizin Berlin, Germany published online $\quad 26.03 .2021$

\section{Bibliography}

Exp Clin Endocrinol Diabetes 2021; 129: S9-\$19

DOI 10.1055/a-1284-6011

ISSN 0947-7349

(C) 2021. Thieme. All rights reserved.

Georg Thieme Verlag KG, Rüdigerstraße 14,

70469 Stuttgart, Germany

German Diabetes Association: Clinical Practice Guidelines Diabetologie 2020; 15 (Suppl 2): S9-S19,

DOI 10.1055/a-1193-3867

\section{Correspondence}

Spokeswoman Gynecology and Obstetrics

Working Group of the Scientific Medical societies,

Berlin, Germany

Prof. Dr. Ute Schäfer-Graf

Berlin Diabetes Center for pregnant women

Department of Gynecology and Obstetrics St. Joseph

Hospital

Wüsthoffstraße 15

12101 Berlin

Germany

ute.schaefer-graf@sjk.de

Speaker Internal Medicine and Diabetology

Working Group of the Scientific Medical societies,

Berlin, Germany

Prof. Dr. Michael Hummel

Diabetological SPP Rosenheim \& Diabetes Research Group,

TU Munich

Max-Josef-Platz 21

83022 Rosenheim

Germany

Michael.Hummel@Irz.uni-muenchen.de 


\section{Preliminary Note}

This practice guideline on gestational diabetes is a practical summary of the evidence-based S3 guideline available on the Internet (https://www.awmf.org/uploads/tx_szleitlinien/057-008I_S3_ Gestationsdiabetes-mellitus-GDM-Diagnostik-Therapie-Nachsorge_2019-06.PDF), published by the German Diabetes Society (Deutsche Diabetes Gesellschaft - DDG) and the German Society for Gynecology and Obstetrics (Deutsche Gesellschaft für Gynäkologie und Geburtshilfe - DGGG), Working Group Obstetrics and Prenatal Medicine (ArbeitsgemAGG).

It replaces the recommendations of DDG and DGGG for the diagnosis and therapy of gestational diabetes dated 2011 and was first published in 03/2018 (planned revision: 03/2019). The primary goal of the guideline is to improve and standardize the prevention, screening, diagnosis, therapy and follow-up care of gestational diabetes through evidence-based recommendations for the outpatient and inpatient sectors.

\section{Definition}

Gestational diabetes mellitus (GDM, ICD-10: O24.4G) is defined as a glucose tolerance disorder first diagnosed in pregnancy with a 75 g oral glucose tolerance test (oGTT) under standardized conditions and quality-assured glucose measurement from venous plasma. The diagnosis is already possible with an elevated glucose level. The definition of manifest diabetes corresponds to that outside a pregnancy; it does not belong to the category of GDM but is referred to as "Diabetes diagnosed during Pregnancy".

\section{Pathophysiology}

The pathophysiology of GDM is largely similar to that of type 2 diabetes. GDM represents a variant of pre-type 2 diabetes and can today be described as a chronic dysfunction characterized by increasing insulin resistance with decreasing $\beta$-cell compensation. Adverse pregnancy outcomes in women with GDM can be mitigated or prevented by timely diagnosis and intensive treatment.

\section{Epidemiology}

In Germany, the GDM prevalence in the perinatal statistics was $5.97 \%$ in 2017 (40 450 cases). This represents an increase of $14.6 \%$ compared to 2016.

\section{Prevention}

For the prevention of GDM there are numerous studies on lifestyle changes (change of diet, increase of physical activity, intake of supplements such as myoinositol, vitamin D, probiotics, fish oil) without clear results. However, women with excess weight and obesity should be advised to reduce their weight by adopting a healthy lifestyle as early as the planning stage of pregnancy and continue to maintain it during pregnancy.

\section{Consequences for Mother and Child}

\section{Acute consequences for the mother}

There is an increased risk of urinary tract and vaginal infections with a resulting increase in premature births, pregnancy-induced hypertension, pre-eclampsia, delivery by $\mathrm{C}$-section, shoulder dystocia, high-grade birth injuries, postpartum bleeding requiring transfusion and depression. Independent of GDM, pre-conception obesity $\left(\mathrm{BMI}>30 \mathrm{~kg} / \mathrm{m}^{2}\right)$ per se leads more often to C-sections and large for gestational age (LGA) children.

\section{Long-term consequences for the mother}

Diabetes risk in later life

After GDM, 35-60\% of women develop diabetes within 10 years (7-8-times higher risk than that of glucose tolerant pregnant women). Already in the first year after pregnancy about $20 \%$ of European women show different forms of disturbed glucose metabolism. The risk of conversion to manifest diabetes is increased in pre-conception obese women, Asian women, GDM diagnosis $<24^{\text {th }}$ week of pregnancy, insulin therapy, oGTT value $\geq 200 \mathrm{mg} /$ $\mathrm{dl}(11.1 \mathrm{mmol} / \mathrm{l})$ during pregnancy for 1 hour, $\mathrm{HbA} 1 \mathrm{c} \geq 5.7 \%$ at the time of GDM diagnosis. The incidence of type 1 diabetes in risk groups $5-10$ years after GDM is $2.3-10 \%$.

\section{Cardiovascular risk profile}

Post-GDM women have a higher risk of developing metabolic syndrome. This is associated with a higher risk of cardiovascular disease (coronary heart disease with myocardial infarction, coronary bypass, coronary angioplasty/stenting, stroke, PAOD) at a young age.

\section{Risk of recurring GDM}

Women of Caucasian origin: risk of recurrence 35-50\% for GDM in subsequent pregnancies. Risk factors: obesity (BMI>30 kg/m²), number of pregnancies, GDM diagnosis before $24^{\text {th }}$ week of pregnancy in previous pregnancies, insulin therapy, interval of $<24$ months between pregnancies, weight gain of more than $3 \mathrm{~kg}$ between pregnancies, increased fasting blood glucose 2 months postpartum. Risk increases to $50-84 \%$ for ethnic groups with high risk of diabetes (Asia, Latin America).

\section{Acute consequences for the child}

The increased intrauterine glucose supply leads to increased fetal insulin secretion (fetal hyperinsulinism), deposition of glycogen in the heart muscle, formation of white adipose tissue and reduced fetal surfactant formation. Fetal hematocrit increases as a result of increased intrauterine erythropoietin levels. At birth, diabetic fetopathy with hypoglycemia, respiratory disorders, polycythemia, hypocalcemia, hypomagnesemia and hyperbilirubinemia is manifested to varying degrees.

\section{Long-term consequences for the child}

While it is unclear whether GDM as such is associated with longterm metabolic consequences for a child, factors associated with GDM in particular (maternal as well as paternal obesity, family diet and exercise habits) increase the long-term risk of childhood obesity and the development of impaired glucose tolerance. Interven- 
tions during pregnancy to reduce elevated glucose levels alone are not sufficient to prevent subsequent childhood obesity.

Postnatal measures in the sense of lifestyle optimization (breastfeeding, type of infant and toddler nutrition, encouragement of early exercise) must follow.

\section{Screening and Diagnostics}

\section{Screening for risk of diabetes at first medical appointment in pregnancy}

At the first medical appointment in early pregnancy (before $24^{\text {th }}$ week) pregnant women with increased risk ( $\triangleright$ Table 1) should be examined for the presence of a glucose tolerance disorder or preexisting (previously undetected) diabetes mellitus (type 1 or 2 ). In the case of diabetes-specific symptoms (polyuria, polydipsia, pronounced glucosuria in spontaneous urine), tests should be performed to determine whether previously undetected pre-conception diabetes mellitus is present. For this purpose, reference is made to the annually-updated joint recommendations for the diagnosis of diabetes mellitus issued by the Commission for Laboratory Diagnostics in Diabetology of the German Diabetes Society (Kommission Labordiagnostik in der Diabetologie der Deutschen Diabetes Gesellschaft - DDG) and the German Society for Clinical Chemistry and Laboratory Medicine (Deutschen Gesellschaft für Klinische Chemie und Laboratoriumsmedizin - DGKL) (see DDG homepage:

https://www.deutsche-diabetes-gesellschaft.de/home.html).

Two methods are possible ( $\vee$ Fig. $\mathbf{1}$ ):

- Measurement of fasting glucose. Blood glucose values in venous plasma of $\leq 92 \mathrm{mg} / \mathrm{dl}(5.1 \mathrm{mmol} / \mathrm{l})$ exclude diabetes mellitus and gestational diabetes (GDM). If the blood glucose value in venous plasma is $\geq 92 \mathrm{mg} / \mathrm{dl}(5.1 \mathrm{mmol} / \mathrm{l})$, a second measurement is performed. This must be done on a different day. The blood glucose measurements must meet laboratory standards. The result of the second measurement is decisive; measurements must be above the cut-off value, otherwise the

- Table 1 Independent risk factors for the emergence of GDM in the course of pregnancy.

\begin{tabular}{|l|c|l|}
\hline & OR & $\mathbf{9 5} \% \mathbf{C l}$ \\
\hline Pregnancies with GDM & & \\
\hline previous GDM & 50.4 & $42.1-60.3$ \\
\hline Weight (>69 kg) & 1.02 & $1.01-1.03$ \\
\hline Pregnancies without GDM & & \\
\hline No GDM in previous pregnancy & 0.45 & $0.4-0.5$ \\
\hline Age (compared to 35 years) & 1.08 & $1.07-1.09$ \\
\hline Weight (>69 kg) & 1.03 & $1.03-1.04$ \\
\hline Height (>1.64 m) & 0.94 & $0.93-0.95$ \\
\hline 1st degree relatives with diabetes & 2.5 & $2.2-2.8$ \\
\hline 2nd degree relatives with diabetes & 1.7 & $1.4-2.1$ \\
\hline Ovulation induction & 1.6 & $1.1-2.3$ \\
\hline Origin: East Asian region & 2.9 & $2.2-3.8$ \\
\hline Origin: South Asian region & 2.3 & $1.8-2.8$ \\
\hline Z-score of birth weight of former children & 1.25 & $1.1-1.3$ \\
\hline
\end{tabular}

diagnosis cannot be made. According to IAPDSG and WHO, blood glucose values of 92-125 mg/dl (5.1-6.9 mmol/l) indicate GDM in early pregnancy. Nutritional counseling and blood glucose self-monitoring are recommended. At plasma glucose levels $\geq 126 \mathrm{mg} / \mathrm{dL}$ ( $7.0 \mathrm{mmol} / \mathrm{l})$ diabetes mellitus is present (likely pre-conception diabetes mellitus).

- Measuring the HbA1c value. With an HbA1c value of $\leq 5.9 \%$ diabetes mellitus-but not early GDM-is excluded, therefore an additional fasting blood glucose determination is necessary. For HbA1c values of 5.9-6.4\% an oGTT is recommended for further clarification with assessment according to IADPSG and $\mathrm{WHO}$. Values $\geq 6.5 \%$ indicate diabetes.

In case of a negative test in early pregnancy, a regular GDM screening according to maternity guidelines is performed in the period from 24 th week +0 to 27 th week +6 , preferably with a $75-g-o G T T$ ( Fig. 1).

\section{Screening for GDM in the period 24th week + 0 to 27 th week +6}

According to the available evidence, screening for GDM should preferably be performed as a one-stage procedure with a 75-g-oGTT between 24 th week +0 and 27 th week +6 in all pregnant women ( Fig. 2).

According to the German maternity guidelines, a primary screening for GDM can be performed with a 50-g-Glucose Challenge Test (GCT) ( Fig. 3) The 50 -g-GCT is performed in a nonfasting state, independent of time of day and food intake. $50 \mathrm{~g}$ of glucose is drunk in $200 \mathrm{ml}$ of water. A blood glucose value in venous plasma of $\geq 135 \mathrm{mg} / \mathrm{dl}(7.5 \mathrm{mmol} / \mathrm{l})$ after one hour is considered positive and requires a subsequent diagnostic 75-g-oGTT. A blood glucose value in venous plasma $\geq 200 \mathrm{mg} / \mathrm{dl}(11.2 \mathrm{mmol} / \mathrm{l})$ is diagnosed as GDM and the 75-g-oGTT is omitted.

According to the HAPO study, $33 \%$ of women with GDM only had an increase in fasting value, which is not detected by the $50-\mathrm{g}$ GCT. However, since the fasting value is the most closely correlated with an unfavorable pregnancy outcome, it is recommended that fasting blood glucose be additionally determined in case of a negative 50 -g-GCT between 24th week +0 and 27 th week +6 of gestation. This is not part of the maternity policy.

GDM screening procedures such as urine glucose, fasting glucose, casual plasma glucose or $\mathrm{HbA} 1 \mathrm{c}$ are not recommended in this guideline; screening using fasting glucose has established itself in Switzerland (see $>$ Fig. 2).

\section{Diagnostics of GDM by means of $75 \mathrm{~g}-0 \mathrm{OGTT}$}

The 75-g-oGTT is performed under standard conditions in the morning on an empty stomach. If the intended time window (24th week +0 and 27 th week +6 ) is exceeded, the test can be performed at a later time according to the medical team's instructions. If there are indications of GDM (polyhydramnios, macrosomia with abdominal circumference $(A C)>$ head circumference $(H C)$ or severe glucosuria), there is also an indication to retest for GDM with a diagnostic 75-g-oGTT in the third trimester in case of a negative screening in the 24-28 weeks of gestation. 


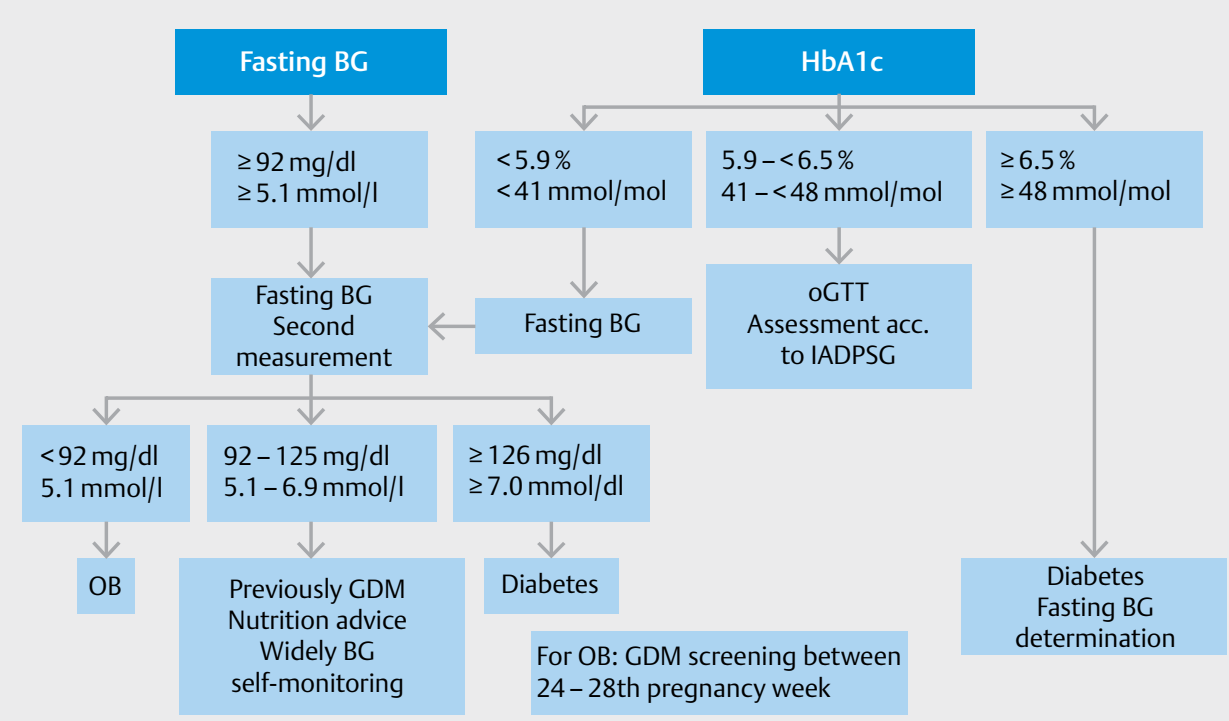

- Fig. 1 Diabetes screening in early pregnancy at risk for DM (HbA1c) or GDM (HbA1c). Source: Gestational diabetes mellitus (GDM), diagnostics, therapy and follow-up care. Guideline of DDG and DGGG-AGG. (S3 level, AWMF registry number: 057-008, March 2018) [rerif]

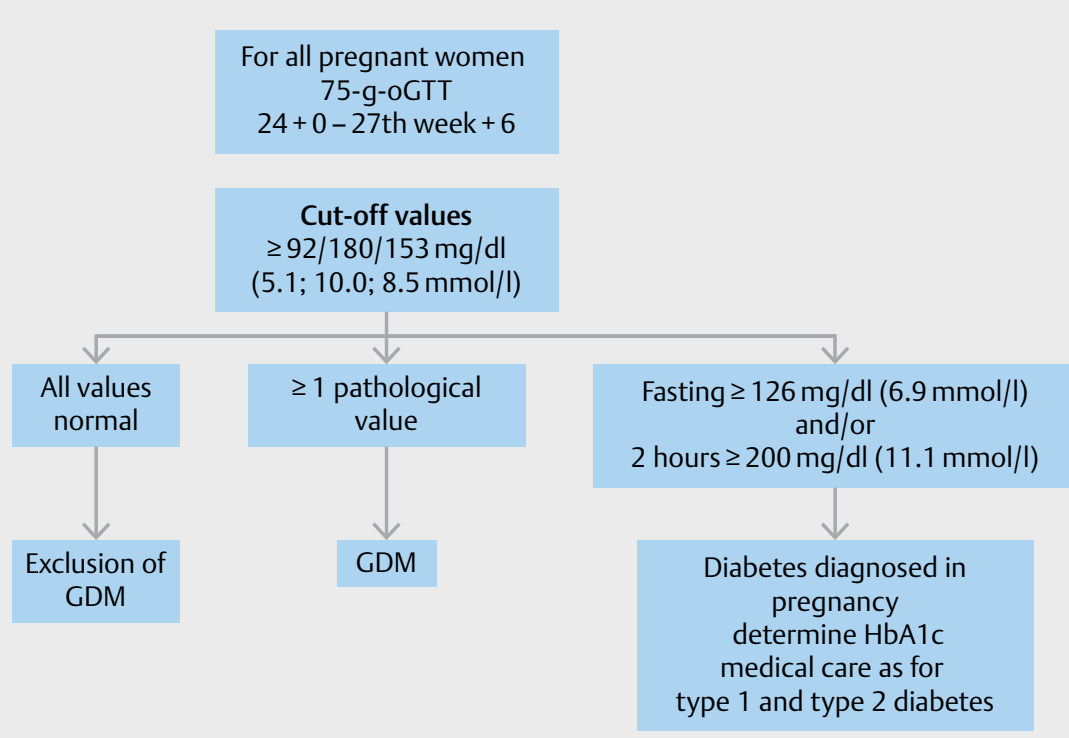

- Fig. 2 Evidence-based screening and diagnostic procedure. Source: Gestational diabetes mellitus (GDM), diagnostics, therapy and follow-up care. Guideline of the DDG and DGGG-AGG. (S3-Level, AWMF Registry Number: 057-008, March 2018) [rerif]

The standard conditions include:

- No acute illness/fever/hyperemesis/medically prescribed bed rest.

- No intake or parenteral administration of drugs with insulinantagonistic effects in the morning before the test (e.g. glucocorticoids, L-thyroxine, $\beta$-mimetics, progesterone). After induction of fetal lung maturity with betamethasone due to the risk of premature birth, at least 5 days must elapse after the last injection and the pregnant woman must be at least partially mobilized before the oGTT is scheduled.
- No previous surgery on the upper gastrointestinal tract (e.g. bariatric surgery with ablative-malabsorptive procedures) -Alternative: Individual blood glucose measurements, especially fasting.

- No unusual physical strain before the test.

- Normal, personal eating and drinking habits with the usual amount of carbohydrates in the last 3 days before the test (the pregnant woman should not prepare for the test by changing her diet, especially by omitting carbohydrates).

- On the evening before the test from 10:00 p.m. on, 


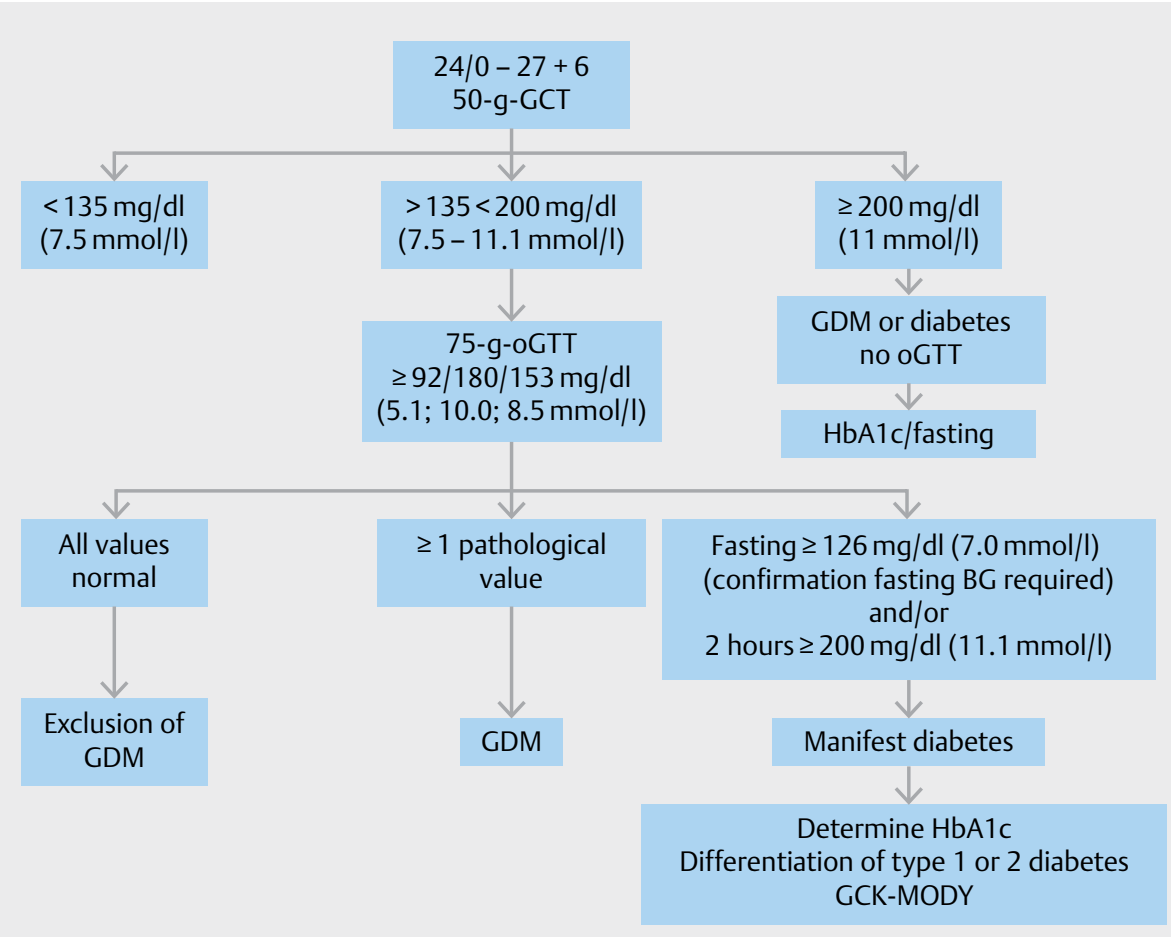

- Fig. 3 Screening for gestational diabetes in the 3rd trimester according to German maternity guidelines Source: Gestational diabetes mellitus (GDM), diagnostics, therapy and follow-up care. Guideline of DDG and DGGG-AGG. (S3 level, AWMF registration number: 057-008, March 2018) [rerif]

- a fasting period of at least 8 hours must be observed.

- The test should not start before 6:00 a.m. and not after 9:00 a.m. the following morning (daily glucose tolerance dependency).

- During the test, the pregnant woman should sit close to the test laboratory and not move unnecessarily.

- Smoking is not allowed before and during the test.

Immediately prior to the start of the test, venous fasting plasma glucose is measured. The pregnant woman then drinks $75 \mathrm{~g}$ anhydrous glucose dissolved in $300 \mathrm{ml}$ water or a comparable oligosaccharide mixture in sips within 3-5 min. Further glucose measurements: one and two hours after finishing drinking the glucose solution. In case of severe morning sickness or vomiting, the test must be postponed by a few days.

Cut-off values for evaluation of the test results of the 75-g-oGTT

The IADPSG criteria are uniformly used as diagnostic threshold values according to the maternity guidelines. If at least one of the three limits in venous plasma is reached or exceeded, GDM is diagnosed ( $\bullet$ Table 2 ).

A fasting blood glucose value $\geq 126 \mathrm{mg} / \mathrm{dl}(7.0 \mathrm{mmol} / \mathrm{l})$ is considered manifest diabetes mellitus.

To confirm, a second measurement of fasting blood glucose on another day and of $\mathrm{HbA} 1 \mathrm{c}$ is indicated. The diagnosis of a manifest diabetes mellitus is confirmed or excluded by the second blood glu-
- Table 2 Cut-off values in venous plasma according to IADPSG consensus recommendations.

\begin{tabular}{|l|c|c|}
\hline $\begin{array}{l}\text { Time point } \mathbf{2 4}^{\text {th }} \text { week }+\mathbf{0} \text { to } \\
\mathbf{2 7}^{\text {th }} \text { week }+\mathbf{6}\end{array}$ & $\begin{array}{l}\text { Cut-off values IADPSG } \\
\text { venous plasma (mg/dl) }\end{array}$ & $\mathbf{( \mathbf { m m o l } / \mathbf { l } )}$ \\
\hline Fasting & 92 & 5.1 \\
\hline After 1 hour & 180 & 10.0 \\
\hline After 2 hours & 153 & 8.5 \\
\hline
\end{tabular}

cose measurement, both values must be $\geq 126 \mathrm{mg} / \mathrm{dl}$. A continuation of the 75-g-oGTT may not be performed if a manifest diabetes mellitus is confirmed by the second blood glucose measurement on an empty stomach $\geq 126 \mathrm{mg} / \mathrm{dl}$.

A value of $\geq 200 \mathrm{mg} / \mathrm{dl}(11.1 \mathrm{mmol} / \mathrm{l})$ after two hours also confirms the diagnosis of diabetes mellitus. An additional HbA1c measurement is then useful. Further care is then provided in the same way as for pre-conception already-known type 1 or type 2 diabetes.

According to HAPO, if the oGTT is limited to the duration of one hour (measurement on an empty stomach and after 1 hour), $2.1 \%$ of all pregnant women with GDM are not detected ( $\bullet$ Table 2 ).

Blood samples and measurement quality requirements For GDM diagnostics, blood glucose values are measured exclusively in venous plasma directly or in venous whole blood and converted into venous plasma values with a factor of $1.11(+11 \%)$ or a plasma-calibrated measuring system is used. Hand-held devices for capillary blood glucose measurement should be not be used. Con- 
version of capillary measured values into venous values is not permitted. Blood glucose measurements for GDM diagnostics must meet the requirements for measurement quality according to the guidelines of the German Medical Association (Richtlinie der Bundesärztekammer - Rili-BÄK). When using unit-use reagents and associated measuring systems, these - as well as all other glucose measuring systems - should be expressly intended for medical use for diagnostic purposes according to the manufacturer's recommendations for the initial diagnosis of manifest diabetes in pregnancy or gestational diabetes. An external quality assurance according to Rili-BÄK guidelines should also be carried out for use in medical private practice.

For detailed information on pre-analytics, methodology and evaluation for the determination of plasma glucose and HbA1c, please refer to the DDG's recommendation "Definition, classification and diagnosis of diabetes mellitus" [1].

Blood glucose measurement: errors, interference factors Venous whole blood or plasma measurements can be erroneous, especially due to different pre-analytical procedures. The main problem is insufficient preanalytical glycolysis inhibition during sample shipment. For practical reasons, the immediate, close-topatient glucose measurement from the venous whole blood sample should be carried out with a Rili-BÄK quality-assured measurement method using a point-of-care measurement system approved for diagnostics (convert to plasma equivalents with a factor of 1.11 ( $+11 \%)$ or use an appropriately plasma-calibrated device; the collection vessel must contain anticoagulants). When shipping venous whole blood samples, the collection vessel should contain not only an anticoagulant and $\mathrm{NaF}$ but also the immediately-effective glycolysis inhibitor citrate/citrate buffer. When filling the vessels, the manufacturer's instructions must be observed in order to obtain valid measurement results.

\section{Diagnostics of GDM after bariatric surgery}

GDM diagnosis via an oral glucose tolerance test is not possible (dumping phenomenon) after surgical procedures that influence resorption. Therefore, to clarify hyperglycemia requiring treatment, monitoring of fasting and 1-hour postprandial blood glucose levels (2-hour values are not meaningful) with daily blood glucose profiles for 2 weeks under normal dietary conditions, for example in the 12, 24 and 32 weeks of gestation, is recommended. If the target values are then exceeded, appropriate diabetological care is recommended. There are no studies available for this procedure.

\section{Therapy}

\section{First medical consultation after GDM diagnosis}

An in-depth conversation in a low-fear atmosphere. The pregnant woman is confronted with the term "diabetes" for the first time. In order to meet the needs of migrant women or illiterate women, suitable interpreters or helpers are called in to ensure that the planned measures are understood and can be implemented. If the pregnant woman is classified as unable to give consent, education and treatment should be postponed.
The elements of the structured initial medical consultation include:

- Importance of the diagnosis for the child and the mother,

- Time frame of the measures to be implemented and the structure of the medical care,

- Reference to the mostly out-patient therapy guidance,

- The purpose of blood glucose self-monitoring,

- Necessity of possible nutritional modification and goal of weight development according to the recommendations of the Institute of Medicine,

- Advantages of regular exercise (increasing insulin sensitivity),

- Reasons for the possible use of pharmacotherapy with insulin or metformin as off-label use,

- End the session with an open discussion including questions about worries and fears.

\section{Physical activity}

Regular physical exercise, fitness programs or sports reduce the risk of GDM, especially in pre-conceptional obese women, and improve resilience during pregnancy and childbirth. In addition, the risk of LGA and C-section is reduced and the need for insulin treatment or daily insulin doses is decreased. Sport can be continued during pregnancy, individual consultation with the gynecologist is required, contraindications must be considered. Endurance or strength training can also be started again during pregnancy at a light to medium level. As the simplest form of physical exercise without aids, brisk walking of at least 30 minutes duration should be carried out at least 3 times a week or daily exercise with an elastic band. The physical activity/training should be started already pre-conceptionally or in the first trimester. Short exercise sessions in the first postprandial hour after main meals are beneficial.

\section{Nutritional advice}

The first therapeutic measure is an individual nutrition evaluation and consultation. This should be based on teaching the principles of a balanced, healthy diet for pregnant women. The network "Gesund ins Leben" (Healthy start to life) has developed a template for this purpose (Federal Center for Nutrition - Bundeszentrum für Ernährung [BZfE] of the Federal Agency for Agriculture and Food Bundesanstalt für Landwirtschaft und Ernährung [BLE], www.gesund-ins-leben.de/inhalt/handlungsempfehlungen-29378.html).

Eating habits, daily rhythm, body weight and socio-cultural-religious status are taken into account in order to achieve the following therapeutic goals:

- Near-normal, pregnancy-specific blood glucose targets without ketosis or hypoglycemia,

- The mother's weight gain recommended for pregnancy,

- Normal growth of the fetus.

The diet should be adapted to the nutrient requirements of pregnancy and be calorically adequate. Recommended distribution of nutrients:

- Carbohydrates: $40-50 \%$.

- Protein: $20 \%$

- Fat: $30-35 \%$. 
Limiting the carbohydrates to $40-45 \%$ daily energy calories lowers postprandial blood glucose levels. However, the carbohydrate percentage should not fall to below $40 \%$ or $175 \mathrm{~g} /$ day. Carbohydrates with a high fiber content and a low glycemic index should be preferred. The recommendation is for the carbohydrates to be divided into 3 not too large main meals and 2-3 smaller snacks (including one late meal) throughout the day, which may help to avoid insulin therapy. There is no evidence of this from RCTs. The amount of carbohydrate should be lower at breakfast than at lunch and dinner (highest blood glucose increase). A late meal containing 1 carbohydrate unit prevents excessive ketone body formation during the night. Sufficient supply of vitamins and minerals (folic acid, vitamin B complex, calcium, vitamin D, magnesium, iron, iodine) should be ensured. Energy-free sweeteners (e.g. aspartame) can be used during pregnancy, taking into account the acceptable daily dosages.

\section{Recommended weight gain}

Weight gain is also based on the pre-conceptional BMI. Weight gain within the IOM limits should be aimed for. A weight loss of $1-2 \mathrm{~kg}$ in the first weeks after a change in diet can occur and is harmless. Controlled weight gain improves the glucose metabolism/increases insulin sensitivity.

An increased pre-conceptional BMI which then exceeds the specified weight limits increases the rate of pregnancy complications (pre-eclampsia, C-section, SGA and LGA children). Falling below these limits increases the rate of fetal growth retardation. In obese women, falling below the limits ( $0-5 \mathrm{~kg}$ total weight gain) is safe. Pregnant women should check and document their weight weekly without clothing in the mornings on an empty stomach at home ( $\triangleright$ Table 3).

\section{Blood glucose monitoring}

Individual blood glucose measurements

Measurement frequency at the beginning: For 1-2 weeks 4-point profile - fasting in the morning and 1 or 2 hours after the start of main meals. If all values within the first 2 weeks are within the target range, the following is reduced to a single daily measurement in rotation or a 4 -point profile $2 \times /$ week. Additional targeted measurements according to the medical team's instructions are possible. Insulin therapy: daily measurements with a 4-point profile or only daily control of the value to be optimized by insulin therapy. Frequency and timing of self-monitoring are continuously adjusted to the effort and course of the therapy and according to the measured results in individual cases. The focus is on minimizing the burden on pregnant women and limiting self-monitoring to the minimum relevant for making a decision. The blood glucose selfmonitoring of pregnant women (control of self-monitoring) as well the accuracy of the blood glucose measuring system (control of the control system) should be checked regularly. Pregnant women with GDM receive suitable diaries for documentation; they are shown how to document correctly.

\section{Continuous glucose monitoring system (CGMS)}

CGMS is not part of the routine care of pregnant women with GDM and does not improve the outcome of the pregnancy.
- Table 3 Recommended range of weight gain during pregnancy.

\begin{tabular}{|c|c|c|}
\hline $\begin{array}{l}\text { Preconceptual } \\
\text { BMI (kg/m²/WHO) }\end{array}$ & $\begin{array}{l}\text { Total weight gain dur- } \\
\text { ing pregnancy }(\mathrm{kg})\end{array}$ & $\begin{array}{l}\text { Weight gain/week } \\
2^{\text {nd }} \text { and } 3^{\text {rd }} \text { Trimester }^{1} \\
\text { (kg) }\end{array}$ \\
\hline 18.5 & $12.5-18$ & $0.5-0.6$ \\
\hline $18.5-24.9$ & $11.5-16$ & $0.4-0.5$ \\
\hline $25.0-29.9$ & $7-11.5$ & $0.2-0.3$ \\
\hline$\geq 30$ & $5-9$ & $0.2-0.3$ \\
\hline
\end{tabular}

- Table 4 Blood glucose setting targets based on plasma-calibrated selfmonitoring devices.

\begin{tabular}{|l|l|l|}
\hline \multirow{2}{*}{ Time } & \multicolumn{2}{|l|}{ plasma equivalent } \\
\cline { 2 - 3 } & $\mathbf{m g} / \mathbf{d l}$ & $\mathbf{m m o l} / \mathbf{I}$ \\
\hline Fasting, preprandial & $65-95$ & $3.6-5.3$ \\
\hline 1 h postprandial & $<140$ & $<7.8$ \\
\hline 2 h postprandial & $<120$ & $<6.7$ \\
\hline
\end{tabular}

HbA1c

Within the scope of early screening ( $\leq 24^{\text {th }}$ week), the HbA1c value is used to diagnose a pre-existing glucose metabolism disorder/ manifest diabetes mellitus (see chapter "Screening for risk of diabetes at first medical appointment in pregnancy"). After a confirmed GDM diagnosis, the HbA1c value is only determined if diabetes mellitus (type 1 or 2 ) is suspected. HbA1c is not relevant for monitoring or therapy control of gestational diabetes. In individual cases, however, it can be helpful as an additional parameter, e.g. in cases of non-compliance for BG self-monitoring or if the quality of the measurements is questionable.

\section{Blood glucose target values}

The blood glucose target values based on plasma-calibrated selfmonitoring devices are shown in $>$ Table 4 . There are no preferences for postprandial measurement after either one or two hours, but the 1-hour values better correlate with fetal growth and are more practical in everyday life. Once a procedure has been established, it must be retained. The at-first limited experience of pregnant women with blood glucose measurements should be taken into account. Blood glucose self-monitoring by pregnant women should not be the sole criterion for therapy control. The accuracy of the pregnant woman's own blood glucose measurements must be checked regularly during the treatment process. The blood glucose target values are indicative; no adverse effects on children can be deduced from occasional exceeding of these targets.

\section{Insulin therapy}

General indication for insulin therapy

If the metabolic goals cannot be reached after making maximum use of lifestyle measures (nutritional therapy, physical activity), there is an indication for insulin therapy. This will generally be given within 2 weeks, although depending on the level of blood glucose values, this may be necessary immediately or only in the course of pregnancy. Fasting blood glucose values $\geq 110 \mathrm{mg} / \mathrm{dl}(6.1 \mathrm{mmol} / \mathrm{l})$ can hardly be influenced by diet during pregnancy. In case of repeated fasting glucose values of $\geq 110 \mathrm{mg} / \mathrm{dl}(6.1 \mathrm{mmol} / \mathrm{l})$, imme- 
diate insulin therapy should therefore be considered. About $20-30 \%$ of pregnant women with GDM require insulin. The indication for insulin therapy is constantly being reviewed. Insulin therapy is indicated if within one week $\geq 50 \%$ of the self-measurements from the 4-point profiles are above the target values. This also applies if $50 \%$ of all of the isolated fasting glucose values (start with basal insulin) or the postprandial values after a meal, most often breakfast (short-acting insulin), are exceeded.

Before starting insulin therapy, the correct implementation of nutrition therapy should be checked once again. The indication should be checked carefully and strictly, since insulin therapy not only causes stress for the pregnant woman, but also significant obstetric effects such as needing to be induced at the due date. Insulin adjustment can usually be started on an outpatient basis.

Insulin therapy with consideration of fetal growth in ultrasound

The effects of maternal hyperglycemia on the fetus vary from individual to individual and are associated with different risks depending on growth patterns. Therefore, the growth of the fetal abdominal circumference (AC) should be taken into account when determining the indication for insulin therapy by promptly obtaining ultrasound findings relevant for decision-making prior to the start of therapy (modified target value concept). A modification of the blood glucose target values depending on the growth pattern of the fetus should help to avoid both over- and under-therapy. Insulin therapy should be started more quickly and slightly lower blood glucose target values should be aimed for in asymmetric macrosomia with a fetal $A C \geq 75$ th percentile, especially in the presence of other risk factors for fetal macrosomia (BMI>30 kg/m²), previous birth of an LGA newborn, fasting BG $>110 \mathrm{mg} / \mathrm{dl}$ in the daily profile at the start of therapy. During pregnancy, fetal growth parameters in ultrasound should also be taken into account when interpreting the measured blood glucose self-monitoring values and the consequences of therapy. In the case of normosomal development of the fetus in the $<75$ th percentile of AC, slight exceedances of the target values are tolerable and the indication for insulin should be rather strict.

\section{Special therapeutic issues}

Isolated elevated fasting glucose levels

Fasting glucose seems to have a strong influence on neonatal complications, LGA and pre-eclampsia. After making maximum use of exercise and nutritional therapy, insulin adjustment should be performed if $>50 \%$ of isolated fasting glucose levels are above the current limit of $95 \mathrm{mg} / \mathrm{dl}(5.3 \mathrm{mmol} / \mathrm{l})$.

Late diagnosis of GDM - insulin therapy close to the due date

Starting therapy with insulin may still make sense even close to a due date in order to avoid neonatal hypoglycemia.

Detecting a glucokinase gene mutation (GCK-MODY)

In about $2 \%$ of all cases of a glucose tolerance disorder in pregnancy, a glucokinase gene mutation (GCK-MODY, MODY 2) with autosomal dominant inheritance is detected. MODY 2 diabetes should be considered in cases of persistently-elevated fasting blood glucose levels in the mother of 99-144 mg/dl (5.5-8.8 mmol/I), a low blood glucose increase in oGTT $<83 \mathrm{mg} / \mathrm{dl}$ ( $<4.6 \mathrm{mmol} / \mathrm{I})$, a normal or only slightly elevated $\mathrm{HbA} 1 \mathrm{c}$ level and a positive family history of "mild" type 2 diabetes over 3 generations.

The suspected diagnosis of a GCK-MODY is confirmed by a genetic analysis (clarification and written consent according to the German Genetic Diagnostics Act - Gendiagnostik-Gesetz is required). Pregnant women with a fasting blood glucose value $>99 \mathrm{mg} / \mathrm{dl}(5.5 \mathrm{mmol} / \mathrm{l})$ in combination with a pre-conceptional $\mathrm{BMI}<25 \mathrm{~kg} / \mathrm{m}^{2}$ should be tested.

Only fetuses of pregnant women with GCK mutation who are not carriers of the GCK mutation have an increased risk of macrosomia and subsequent complications of increased maternal glucose during pregnancy. Insulin therapy should only be initiated in fetuses of pregnant women with GCK mutation if disproportionate growth ( $A \cup \geq 75$. percentiles) has been detected in ultrasound.

Implementation of insulin therapy

The indication for insulin should be checked for the first time within 1-2 weeks after the start of the basic therapy (diet, exercise), taking into account blood glucose self-monitoring, blood glucose laboratory tests and mother and fetus biometric data, and then continuously during the treatment process as determined by the medical team. Insulin should be set according to the ICT principle, but only basal or short-acting insulin may be required. If short-acting human insulins cannot reduce postprandial blood glucose levels in a targeted manner with sufficient dosage, a switch to insulin aspart or lispro should be considered. Both rapid-acting and longacting insulin analogues can also be mainly used. Insulin adjustment should generally be started on an outpatient basis and should be reserved for experienced diabetologists and perinatal physicians with appropriate specialties in the care of diabetic pregnant women.

\section{Oral antidiabetics and GLP-1 agonists}

After therapeutic clarification of off-label use, the administration of metformin can be considered in pregnant women with GDM and suspected pronounced insulin resistance (insulin requirement $>1.5$ $\mathrm{IU} / \mathrm{kg}$ KG) as well as according to individual indications. Before beginning with metformin, internal contraindications must first be clarified, and laboratory parameters, e.g. serum creatinine and creatinine clearance, must first be determined. A metformin daily dose of $2.0 \mathrm{~g}$ should not be exceeded. Sulfonylurea preparations should not be used during pregnancy. Alpha-glucosidase inhibitors, glitazones, glinides, DPP-4 inhibitors and GLP-1 agonists should not be prescribed to pregnant women with GDM due to lack of approval, lack of experience and insufficient studies.

\section{Obstetrical Care}

\section{Fetal monitoring}

Fetal monitoring depends on additional risk factors and the severity of maternal hyperglycemia. 


\section{Ultrasounds}

\section{$1^{\text {st }}$ trimester}

In case of GDM diagnosed before 14 weeks of gestation with additional risk factors (elevated blood glucose and HbA1c levels, history of heart malformations, obesity) or GDM diagnosed earlier, early detailed ultrasound organ diagnosis and echocardiography should be performed to exclude severe malformations of the fetus in the 11 th $+0-13$ th +6 weeks of gestation. Requirements of DEGUM Level II.

\section{$2^{\text {nd }}$ trimester}

In case of GDM with a diagnosis before 24 weeks of gestation and additional risk factors (elevated blood glucose and $\mathrm{HbA} 1 \mathrm{c}$ values, anamnestic heart malformations, obesity), a differentiated further organ diagnosis with echocardiography of the fetus should be performed in the 19th-22nd weeks of gestation.

\section{$3^{\text {rd }}$ trimester}

Biometry is performed at 2 to 3 -week intervals (AC percentiles), in case of abnormal growth (macrosomia, IUGR) at correspondingly shorter intervals. Before delivery: weight estimation and assessment of the ratio of abdomen to head is recommended (fetal macrosomia is a risk factor for shoulder dystocia). The extent of subcutaneous fetal fat tissue can be included in the assessment.

\section{Doppler ultrasound}

The usual indications for all pregnancies apply. Doppler ultrasound examinations are not indicated solely because of the GDM diagnosis.

\section{Cardiotocography (CTG)}

- For nutritional therapy: weekly CTG checks from the 36th week of pregnancy with a frequency adapted to the individual situation.

- With insulin therapy: as for a pregnant woman with pre-conceptionally-known type 1 diabetes, in accordance with the guideline for the care of diabetic pregnant women (AWMF guideline 057/023), CTG checks from 32nd week onward with a frequency adapted to the individual situation.

Antepartum monitoring of the mother

- Increased pre-eclampsia risk: intensive therapy of GDM significantly reduces the risk.

- Increased risk of intrauterine death: if not treated or with poor blood glucose control

\section{Preterm birth (induction of fetal lung maturity, tocolytics)}

Betamethasone for induction of fetal lung maturity (before 34 th +0 week): blood glucose levels increase, therefore strict indication. Adjust the insulin dose individually or initiate insulin treatment from a blood glucose value $\geq 200 \mathrm{mg} / \mathrm{dl}$ ( $11.1 \mathrm{mmol} / \mathrm{l}$ ) or hyperglycemic symptoms.

Administer tocolytics preferably with an oxytocin or calcium antagonist and not with a beta-agonist (increase in maternal blood glucose, intensified by simultaneous bed rest).

\section{Birth planning}

Birth - Choice of maternity hospital

Pregnant women with GDM are high-risk pregnant women.

- GDM with nutritional therapy: delivery in a maternity hospital with diabetological experience and affiliated neonatology should be advised.

- GDM with insulin therapy: in order to ensure optimal primary care of the child, the delivery must be carried out in a maternity hospital with an affiliated neonatology department (perinatal center level 1 or 2 ) and the delivery must be in accordance with guidelines.

Time of birth, indication for initiation Inducting $<39$ th +0 week increases neonatal morbidity and transfer rate and should be avoided. An induction in the 39+0-39th + 6 week may be considered but is associated with a $50 \%$ increase in the rate of inducing and does not reduce neonatal morbidity. Premature (before 38 th +0 weeks of gestational age) inducing due to poor BG adjustment should not be attempted due to the morbidity associated with preterm delivery. Prenatal optimization of blood glucose control should be sought. There is evidence that fetal morbidity in insulin-dependent GDM can be reduced by inducing at 40 weeks gestation; it should therefore be offered. For GDM and ultrasonographic fetal estimated weight $>95$ th percentile, possible benefits of inducing at 37-0 weeks of gestation should be weighed against the effects of earlier gestational age at birth.

C-section delivery

- With an estimated birth weight of $\geq 4500 \mathrm{~g}$, the risk of shoulder dystocia increases significantly: a planned C-section should be recommended.

- Estimated weight 4000-4499g: pregnant women with an individually increased risk of shoulder dystocia according to fetal biometry, especially with a pronounced head/abdomen difference, should be informed of this risk. However, the pregnant woman should be aware of the inaccuracy of the estimate, which increases with increasing birth weight, the risk of C-section and the consequences for placental nidation disorders in the following pregnancy.

\section{Pregnant women after bariatric surgery}

Pregnant women after bariatric surgery are considered high-risk pregnant women and must be closely monitored by obstetrics. Attention must be paid to sufficient substitution according to the increased need.

\section{Postpartum Support}

\section{Glucose control of the mother during birth and in the postpartum phase}

If the birth is induced, short-acting insulins should be used for better control.

Blood glucose target during birth in capillary plasma: between 90 and $140 \mathrm{mg} / \mathrm{dl}(4.4-7.2 \mathrm{mmol} / \mathrm{l})$.

- Nutritional therapy (well adjusted): routine maternal blood glucose control during birth is not required. 
- Insulin therapy: measurement of blood glucose levels for GDM insulin every two hours, adjusting the time intervals individually as needed; in the case of GDM, insulin is rarely needed during birth. Insulin therapy is terminated postpartum. Further control by means of a 4-point daily profile on the 2nd day postpartum, inform diabetologists in case of repeatedly high values. The same limits apply as for non-pregnant women. Insulin is indicated postpartum for blood glucose levels $\geq 200 \mathrm{mg} / \mathrm{dl}$ ( $11.1 \mathrm{mmol} / \mathrm{l}$ ) or hyperglycemic symptoms.

\section{Follow-up care of the mother}

After pregnancy, the glucose tolerance disorder does not regress in about $13-40 \%$ of cases. There is a 7 - to 8 -fold increased risk of diabetes for women after GDM. The risk is particularly increased in the case of pre-conceptional obesity, positive family history of diabetes mellitus, insulin requirements for GDM, advanced age, Asian and black African women.

\section{Postpartum $75 \mathrm{~g}$ oGTT}

Postpartum normal blood glucose levels: 6-12 weeks after birth 75-g-oGTT independent of breastfeeding. Normal values for oGTT outside pregnancy with fasting blood glucose measurements and 2 hours in venous plasma after eating according to WHO guidelines:

- Normal: fasting $<100 \mathrm{mg} / \mathrm{dl}(5.6 \mathrm{mmol} / \mathrm{l}), 2 \mathrm{~h}$ after eating $<140 \mathrm{mg} / \mathrm{dl}(7.8 \mathrm{mmol} / \mathrm{l})$,

- Diabetes mellitus: fasting $\geq 126 \mathrm{mg} / \mathrm{dl}(7.0 \mathrm{mmol} / \mathrm{l})$ and or $2 \mathrm{~h}$ after eating $\geq 200 \mathrm{mg} / \mathrm{dl}(11.1 \mathrm{mmol} / \mathrm{l})$,

- Impaired fasting glucose (IFG): $100-125 \mathrm{mg} / \mathrm{dl}$ (5.6$6.9 \mathrm{mmol} / \mathrm{l})$,

- Impaired glucose tolerance after 2 h (IGT): 140-199 mg/l (7.8-11.05 mmol/l).

Primary determination of $\mathrm{HbA} 1 \mathrm{c} 6-12$ weeks postpartum is not recommended for diagnostic purposes, even fasting glucose alone is not sufficient. In the presence of impaired glucose tolerance or other risks such as pre-conception obesity or GDM insulin therapy, women are given intensive advice on lifestyle measures to reduce the risk of conversion to manifest diabetes.

\section{Further postpartum controls}

The increased risk for women with GDM to develop postpartum diabetes within the next 10 years requires continuous follow-up with glucose metabolism controls. The recommendations for diabetes diagnosis of the National Care Guideline Therapy of Type 2 Diabetes (Nationalen Versorgungs-Leitlinie Therapie des Typ-2-Diabetes) [2] apply, usually with a fasting glucose and HbA1c, if necessary oGTT every 2 years. After postpartum diagnosis of impaired fasting glucose (IFG) or impaired glucose tolerance (IGT), a yearly oGTT is recommended. When planning a pregnancy, at least one HbA1c and one fasting glucose should be used to diagnose diabetes. For every subsequent pregnancy, perform an early hyperglycemia diagnostic in the first trimester (first medical appointment) according to this guideline. In case of development of type 1 diabetes (women with a BMI $<30 \mathrm{~kg} / \mathrm{m}^{2}$ and insulin therapy of GDM): au- toantibody screening (e.g. anti-GAD, anti-IA2, anti-ICA and antiZnT8).

\section{Peripartum depression}

Compared to glucose-tolerant pregnant women, the rate of postpartum depression in women with GDM, especially from socially deprived backgrounds, is up to twice as high. The Edinburgh Postnatal Depression Scale (EPDS) in German is a suitable tool for searching for postpartum depression. According to this, all EPDS sum scores of at least 10 indicate a depressive mood. This suspicion should be further clarified by a specialist in order to initiate therapy in time. The oGTT timeframe of 6-12 weeks after pregnancy is a suitable timeframe to use the well-being questionnaire.

\section{Diabetes prevention}

There is a 7- to 8-times higher risk of diabetes for women after GDM. Lifestyle modification is considered a priority measure. Women with a glucose tolerance disorder after GDM benefit from lifestyle changes to prevent diabetes-related and macroangiopathic complications. They are advised and instructed to follow a diet adapted to their needs, to normalize their weight, to take part in smoking cessation training, if necessary, and to engage in regular physical activity. This can reduce the manifestation of diabetes by $50 \%$ in 3 years and $35 \%$ in 10 years. In addition, women who have not yet completed family planning should be made aware of the risks of unplanned pregnancy with diabetes.

\section{Breastfeeding}

After GDM, mothers breastfeed their children less frequently and for shorter periods than women without diabetes, especially in cases of excess weight and obesity, with insulin-treated GDM and lower educational levels. Mothers with GDM breastfeed more successfully if they have received breastfeeding advice before birth and received both non-medical and medical care postnatally.

\section{Breastfeeding and effects on maternal health}

Breastfeeding has short-term positive effects on maternal metabolism (improvement of glucose homeostasis, insulin sensitivity and lipid metabolism parameters). In addition, breastfeeding seems to significantly reduce the risk of type 2 diabetes and metabolic syndrome in mothers with GDM up to 15 years after delivery.

\section{Breastfeeding and effects on child health}

No or shorter breastfeeding ( $<3$ months) is associated with later childhood obesity, especially in children of obese gestational diabetics.

Women with GDM should therefore be strongly encouraged to breastfeed their children. In particular, obese pregnant women with GDM should be especially motivated and given support in breastfeeding. Recommendation: all pregnant women with GDM should be informed about the advantages of breastfeeding for mother and child by a breastfeeding counselling service before delivery and strategies for a successful start of breastfeeding should be pointed out; exclusive breastfeeding is encouraged for at least 4-6 months. Even after the introduction of complementary foods - at the earli- 
est at the beginning of the 5th month, at the latest at the beginning of the 7th month - infants should continue to be breastfed for as long as possible.

\section{Conflict of Interest}

An overview of conflicts of interest can be found at https://www. awmf.org/fileadmin/user_upload/Leitlinien/057_D_Diabetes_ Ges/057-008i_S3_Gestationsdiabetes-mellitus-GDMDiagnostik-Therapie-Nachsorge_2018-03.pdf

\section{Literature}

[1] Praxisempfehlung „Definition, Klassifikation und Diagnostik des Diabetes mellitus" der DDG Diabetologie und Stoffwechsel. 2017; 12: (Suppl. 2): S94-S100. doi:10.1055/s-0043-115953

[2] Bundesärztekammer, Kassenärztliche Bundesvereinigung, Arbeitsgemeinschaft der Wissenschaftlichen Medizinischen Fachgesellschaften Hrsg. Nationale Versorgungs-Leitlinie Therapie des Typ-2-Diabetes. 1. Auflage 2013: 29-32.

\section{Notice}

This article was changed according to the following Erratum on November $25^{\text {th }} 2021$.

\section{Erratum}

This erratum is being published to remove the authors Vladimir Patchev and Monika Nothacker. 